\title{
CRITÉRIOS ANTROPOMÉTRICOS NO DIAGNÓSTICO DA DESNUTRIÇÃO EM PROGRAMAS DE ASSISTÊNCIA À CRIANÇA
}

Carlos Augusto Monteiro*

MONTEIRO, C. A. Critérios antropométricos no diagnóstico da desnutrição em programas de assistência à criança. Rev. Saúde públ., S. Paulo, 18:209 - 17, 1984.

RESUMO: Examinando a teoria da avaliação do estado nutricional por meio da antropometria e considerando as características e objetivos da assistência primária à saúde infantil, formulam-se orientações gerais para o diagnóstico antropométrico-nutricional a nível de unidades sanitárias.

Considerando a fisiologia do crescimento e as evidências epidemiológicas existentes quanto à história natural de desnutrição em nosso meio, são propostos critérios específicos para diagnóstico de desnutrição e indicação de recuperação nutricional, para avaliação de eficácia de tratamento e para determinaçăo de alta.

UNITERMOS: Crianças, estado nutricional. Antropometria. Desnutrição, diagnóstico.

\section{INTRODUÇÃO}

De há muito, a avaliação do crescimento é considerada importante atividade da assistência primária de saúde. A par de constituirse em sensivel aferidor das condições gerais de saúde do indivíduo, a monitorização do crescimento permite alertar profissionais de saúde e comunidade para a denominada "desnutrição invisível", ou seja, para as formas insidiosas e crônicas da desnutrição infantil. Nos países não desenvolvidos, estas formas assumem proporções de verdadeira endemia, em muito superando a frequiência atribuída aos quadros clínicos bem definidos de desnutrição. Organismos internacionais, empenhados na melhoria das condições de saúde das crianças de todo o mundo, como o
$\mathrm{UNICEF}^{7}$, vêem o adequado acompanhamento do crescimento até os cinco anos de idade, envolvendo ativamente tanto mães como profissionais de saúde, como uma das quatro maiores prioridades atuais da assistência à criança ao lado da imunização, reidratação oral e aleitamento materno.

$O$ presente trabalho propze-se a:

- examinar os pressupostos teóricos do diagnóstico do estado nutricional efetuado por meio da antropometria;

- formular orientações gerais para o diagnóstico antropométrico realizado a nível de unidades sanitárias;

- propor critérios antropométricos específicos para o diagnóstico da desnutrição e para a avaliação da recuperação nutricional nas diversas idades.

* Do Departamento de Nutrição da Faculdade de Saúde Pública da USP - Av. Dr. Arnaldo, 715 - 01255 - São Paulo, SP - Brasil 
MONTEIRO, C. A. Critérios antropométricos no diagnóstico da desnutrição em programas de assistência à criança. Rev. Saúde públ., S. Paulo, 18: 209 - 17, 1984.

\section{PRESSUPOSTOS DA ANTROPOMETRIA NUTRICIONAL.}

Em nosso meio e no conjunto dos países não desenvolvidos, a deficiência nutricional de maior prevalência e de mais amplas consequêencias para a saúde é a desnutrição protéico-calórica (DPC). Em crianças, a deficiência de proteínas e calorias invariavelmente ocasiona retardo na velocidade de crescimento, sendo este retardo, freqüentemente, a primeira manifestação detectável da deficiência. Por outro lado, onde a desnutrição infantil é endêmica, como em nosso meio, outras causas de retardo do crescimento são relativamente menos importantes. Ambos os fatos autorizam o diagnóstico presuntivo da DPC a partir da identificação de déficits de crescimento e, aliados a aspectos de praticidade operacional, tornam a antropometria o método de escolha para o diagnóstico da deficiência em situações como as nossas.

Exame de sinais clínicos e exames bioquímicos, procedimentos de escolha para o diagnóstico de deficiência de micronutrientes (ferro, iodo, vitamina $A$, entre outros), não apresentam a mesma utilidade em relação à deficiência de proteínas e calorias. Nesta deficiência, sinais clínicos como edema e lesões de pele e alterações bioquímicas como hipoalbuminemia ocorrem tardiamente e, ainda assim, apenas nos casos mais severos. Nesta última medida os referidos exames são procedimentos capazes de identificar a severidade da desnutrição.

A anamnese alimentar é instrumento valioso para a determinação da etiologia da DPC, sendo sobretudo útil na orientação das ações terapêuticas de recuperação. Não fornece, entretarito, indicação confiável de diagnóstico. Entre outros motivos, porque a quantificação do consumo alimentar e o estabelecimento das necessidades nutricionais do indivíduo são procedimentos complexos e habitualmente sujeitos a excessiva imprecisão.

Elementos Básicos para o Diagnóstico Antropométrico-Nutricional
O diagnóstico da DPC por meio do acompanhamento do crescimento pressupõe a adequada execução das seguintes etapas preliminares:

a) registro exato de idade e sexo da criança;

b) tomada periódica precisa de medidas de altura e peso;

c) adoção de padrão antropométrico de referência que indique, nas diversas idades e em ambos os sexos, a distribuição esperada para medidas de indivíduos bem nutridos.

Bastante cuidado deve haver na obtenção das informações relativas a idade, altura e peso, pois as mesmas estão sujeitas a várias fontes de erros e estes, ainda que pequenos, podem determinar sensíveis distorções no diagnóstico do estado nutricional.

A tomada de outras medidas está indicada apenas quando operacionalmente não for possível obter altura e peso (caso do perímetro braquial que guarda boa relação com peso $^{4}$ ) ou quando se desejar maior especificação do comprometimento do crescimento (caso do perímetro braquial e da dobra cutânea tricipital que, em conjunto, podem fornecer indicações sobre o comprometimento individualizado dos tecidos muscular e adiposo ${ }^{5}$.

0 padrão de referência teoricamente deveria se confundir com padrão de normalidade dos indivíduos examinados, isto é, deveria ser construído a partir de medidas observadas em indivíduos bem nutridos e com potencial genético de crescimento idêntico ao dos examinados. $\mathrm{Na}$ prática, dadas as diminutas diferenças que parecem separar, pelo menos nos primeiros anos de vida, o crescimento dos diferentes grupos étnicos quando favoráveis às condições ambientais ${ }^{1}$, aceita-se que padrões de referência, desde que construídos fidedignamente e a partir de populações cujo crescimento físico se dê livre de impedimentos ambientais, possam ser utilizados como aproximações válidas do que seriam padrões de normalidade. Nesta última condição encontram-se os padrões "Santo André Classe IV"3 e "NCHS"6, construidos, respectivamente, a partir de famílias de alto nível sócio-econômico do mu. 
MONTEIRO, C. A. Critérios antropométricos no diagnóstico da desnutrição em programas de assistência à criança. Rev. Saúde públ., S. Paulo, 18: 209 - 17, 1984.

nicípio de Santo André e a partir de amostras probabilísticas da população americana.

\section{Identificação de Déficits de Crescimento e Diagnóstico da Desnutrição}

O diagnóstico presuntivo da DPC, por meio do exame antropométrico, baseia-se fundamentalmente no encontro de medidas que, sendo suficientemente baixas, sejam de ocorrência improvável em indivíduos bem nutridos. Para tanto, torna-se necessário definir no padrão de referência valores críticos que delimitem, em cada idade e sexo, medidas capazes de indicar com razoável especificidade a presença da desnutrição.

A escolha dos valores críticos, a partir dos quais se fará o diagnóstico da desnutrição, dependerá do quanto de especificidade se pretende para o diagnóstico. Como a especificidade deste é o inverso da proporção admitida de "falsos positivos" (proporção de indivíduos normais diagnosticados erroneamente como desnutridos), quanto menores os valores críticos adotados, menor será a probabilidade de atribuir desnutrição a indivíduos normais e, portanto, maior será a especificidade do diagnóstico. Vale notar, entretanto, que a sensibilidade do diagnóstico, ou seja, sua capacidade em identificar todos os indivíduos com algum déficit de crescimento, variará na razão inversa da especificidade obtida: maior especificidade significando obrigatoriamente mais "falsos negativos" e, portanto, menor sensibilidade.

A escolha definitiva dos valores críticos será decidida em cada situação específica, considerada a inconveniência advinda da presença de "falsos positivos" e de "falsos negativos". Para um programa de recuperação nutricional, por exemplo, "falsos positivos", em excesso, diluirão demasiadamente os recursos disponíveis e tornarão difícil a avaliação dos resultados obtidos, enquanto "falsos negativos" em excesso significarão a não inclusão no programa de muitos indivíduos que dele poderiam se beneficiar. Outro fator a ser considerado se refere à freqüência esperada para a desnutrição no local: em locais onde esta é elevada, poder-se-ia teoricamente trabalhar com especificidades menores (e, portanto, com sensibilidades maiores) na medida em que a população normal, aquela que á exposta a diagnósticos de "falsos positivos", é relativamente pouco numerosa. Situação inversa ocorreria em locais com pequena prevalência de desnutridos, como nos países desenvolvidos, onde a especificidade do diagnóstico deve ser necessariamente alta sob pena de os "falsos positivos" superarem em número os verdadeiros desnutridos.

Independente de quais sejam ao final os valores críticos adotados para definir o estado de desnutrição, eles deverão garantir que em todas as idades e em ambos os sexos o diagnóstico da desnutrição seja feito com idêntica especificidade. Esta condição é possível quando a definição dos valores críticos é feita a partir de percentis do padrão antropométrico de referência. Assim, a utilização em todas as idades e em ambos os sexos, tanto para altura quanto para peso, de valores críticos que correspondam, por exemplo, ao percentil 10 do padrão, garantirá que o diagnóstico da "anormalidade antropométrica" e, portanto, da presumível desnutrição, sempre será feito com $90 \%$ de especificidade. Isto porque apenas $10 \%$ de indivíduos normais serão esperados naquela situação.

A costumeira definição de valores críticos baseada em afastamentos percentuais em relação à média ou mediana do padrão - tal como preconizado na conhecida "Classificação de Gomez"2 - não garante a necessária condição de idêntica especificidade e nem mesmo permite o conhecimento prévio da especificidade do diagnóstico realizado nas diferentes categorias de idade e sexo. A definição baseada em afastamentos da média quantificados em unidades de desvio-padrão é alternativa aceitável apenas quando é normal a distribuição das medidas no padrão, o que ocorre para altura, mas freqüentemente não ocorre para peso.

\section{Desnutrição Passada e Desnutrição Atual}

O exame antropométrico de um indivíduo em apenas um único momento é sufici- 
MONTEIRO, C. A. Critérios antropométricos no diagnóstico da desnutrição em programas de assistência à criança. Rev. Saúde públ., S. Paulo, 18: 209 - 17, 1984.

ente para que seu crescimento seja avaliado e para que eventuais déficits e, portanto, presumíveis processos de desnutrição sejam identificados. A observação única não situa entretanto o momento de ocorrência do déficit antropométrico, não diferenciando assim processos pregressos de desnutrição de processos recentes e/ou atuais. Tal diferenciação é sobretudo importante na indicação da intervenção para recuperação nutricional, pois que a reversão dos prejuizos determinados pela desnutrição será tanto mais possivel quanto mais precoce for a intervenção.

Com o exame antropométrico periódico, torna-se possivel avaliar o crescimento em períodos individualizados de tempo, sendo assim possível situar o período de origem de um dado déficit e, portanto, a natureza recente ou pregressa do processo de desnutrição que o determinou. Desta maneira, o registro de um déficit de crescimento associado a recentes incrementos normais de altura e peso indicará desnutrição pregressa, enquanto o mesmo déficit associado a incrementos desfavoráveis indicará desnutrição recente. Um terceiro diagnóstico, de desnutrição crônica, seria feito se o déficit de crescimento estivesse associado a sucessivos incrementos desfavoráveis.

$\mathrm{Na}$ ausência de acompanhamento antropométrico, uma alternativa capaz de indicar, de algum modo, a natureza recente ou pregressa da desnutrição seria a avaliação do peso diante da altura da criança. Em virtude de que déficits de altura se estabelecem de forma bastante mais lenta do que déficits ponderais, déficits de peso para altura indicariam desnutrição recente enquanto que déficits proporcionados de altura e peso sugeririam desnutrição pregressa ou desnutri. ção crônica. Esta última diferenciação seria possivel apenas com o acompanhamento do crescimento mencionado anteriormente.

A determinação da natureza da desnutrição, efetuada tanto a partir da avaliação dos incrementos de altura e peso quanto da adequação do peso à altura, deveria ser feita contrapondo-se os resultados observados aos de um padrão de referência, respeitadas as considerações efetuadas anteriormente sobre características do padrão e escolha de valores críticos para definir anormalidade. Em função, entretanto, de que a maioria dos padrões antropométricos de referência não trazem a distribuição de incrementos (por serem geralmente baseados em estudos transversais) nem a distribuição de pesos para altura (o que exige número muito grande de observações), a natureza da desnutrição é freqüentemente determinada de modo menos preciso; no caso de se avaliar incrementos das medidas em um dado intervalo de tempo, comparando-se os mesmos com a progressão correspondente observada nos valores médios do padrão, e no caso de se avaliar a adequação do peso à altura, comparando as adequações obtidas individualmente para altura e para peso.

\section{ORIENTAÇÕES GERAIS PARA O DIGNÓSTICO ANTROPOMÉTRICO EFETUADO A NIVEL DE UNIDADES SANITÁRIAS.}

Tendo em vista as considerações preliminares feitas quanto ao diagnóstico nutricional por meio da antropometria e atentando. se também para características tanto da clientela infantil quanto da assistência usualmente prestada a nível de unidades sanitárias, as seguintes orientações são propostas:

1) a avaliação do crescimento deve ser o instrumento fundamental para identificação da desnutrição, sendo a anamnese alimentar e o exame de sinais clínicos observados no sentido de informar, respectivamente, a etiologia da deficiência e a severidade do quadro;

2) todas as crianças devem ser pesadas (e, se possível, medidas) pelo menos mensalmente no primeiro ano de vida, bimensalmente no segundo, trimestralmente no terceiro e semestralmente nas idades subseqüentes;

3) todas as medidas tomadas devem ser assinaladas em um gráfico de crescimento que contenha duas curvas: uma correspondente aos valores medianos esperados e outra correspondente aos valores an- 
MONTEIRO, C. A. Critérios antropométricos no diagnóstico da desnutrição em programas de assistência à criança. Rev. Saúde públ., S. Paulo, 18: 209 - 17, 1984.

tropométricos críticos que indicarão o limite inferior da normalidade;

4) o padrão antropométrico de referência deve ser o padrão "Santo André Classe IV" em virtude de o mesmo possuir as qualificações necessárias e ser mais conhecido em nosso meio;

5) os valores antropométricos críticos, ou seja, as medidas que determinarão o limite inferior da normalidade em cada idade e sexo devem ser estabelecidos sempre como um dado percentil do padrão de referência ("percentil crítico"), possibilitando, assim, a uniformização da especificificidade do diagnóstico.

Conforme discutido anteriormente, a escolha do percentil crítico deve ser feita em função do propósito buscado pela avalia. ção nutricional, considerada a inconveniência acarretada por "falsos-positivos" e por "falsos-negativos". Se se admite que na assistência infantil prestada em unidades sanitárias o objetivo central da avaliação nutricional é o de identificar crianças desnutridas que deverão merecer cuidados especiais para sua recuperação, é evidente que neste nível devem ser lgualmente valorizadas tanto a especificidade (capacidade de não incluir "falsos-positivos") quanto a sensibilidade (capacidade de não incluir "falsos-negativos") do diagnóstico. Tendo em vista esta condição e considerando que especificidade e sensibilidade variam inversamente à medida que se adotam percentís maiores ou menores, é proposta a orientação:

6) o percentil crítico escolhido deve situar-se eqüidistante a percentis críticos máximo e mínimo que retratem as menores especificidade e sensibilidade que, em cada situação, sejam consideradas ainda aceitáveis pelo programa de assistência à criança.

Esta última proposição, como se verá, implica tomada de decisão onde diferentes fatores devem ser pesados, entre os quais os objetivos gerais do programa de assistência à criança, a cobertura atual e prevista do programa, os custos envolvidos com a recuperação nutricional, os recursos existentes e ainda os recursos que poderão ser mobiliza- dos no futuro.

Como forma de indicar o percentil crítico máximo e, portanto, expressar a especificidade mínima ainda conveniente ao programa, propõe-se a seguinte orientação:

7) o percentil crítico máximo deve ser aquele que determina, na população em ques tão, uma proporção de indivíduos diagnosticados que exceda em certo número mínimo de vezes a proporção de indivi. duos normalmente esperados naquela situação (isto é, a proporção de indivíduos que, mesmo bem nutridos, apresentam medidas iguais ou inferiores às do percentil crítico).

E evidente que quando se decidir por uma relação mínima de 2:1 entre proporção de diagnosticados e proporção esperada, estarse-á assumindo que uma representação de metade de "falsos-positivos" entre o total de diagnósticos efetuados ainda será tolerada pelo programa. E também evidente que quando a decisão for por relações como 3:1 ou 4:1, o programa não estará aceitando que os "falsos-positivos" representem, respectivamente, mais do que um terço ou um quarto do total de diagnósticos. $E$ interessante notar que, nas três situações aventadas, o percentil crítico máximo situar-se-á, respectivamente, entre os percentis 1 e 50,1 e 33 e 1 e 25 . Dentro destes intervalos, o percentil crítico máximo tenderá a valores superiores nas populações mais atingidas pela desnutrição e a valores inferiores nas populações menos atingidas.

Como forma de indicar o percentil crítico mínimo e, portanto, expressar a mínima sensibilidade ainda conveniente ao programa, propõe-se a seguinte orientação:

8) o percentil crítico mínimo deve ser o menor percentil ainda capaz de identificar corretamente determinadas proporções de déficits de crescimento cuja magnitude tenha sido considerada moderada ou severa.

Uma vez definidos o que seriam déficits moderados e severos e definidas as sensibilidades exigidas para déficits como estes, o percentil crítico mínimo poderá ser identificado pela simples inspeção da distribuição 
MONTEIRO, C. A. Critérios antropométricos no diagnóstico da desnutrição em programas de assistência à criança. Rev. Saúde públ., S. Paulo, 18: 209 - 17, 1984.

da medida antropométrica em questão em indivíduos normais, ou seja, pela simples inspeção do padrão antropométrico adotado. A partir da inspeção do padrão "Santo André Classe IV" verifica-se, por exemplo, que na idade de doze meses, sexo masculino, o percentil 5 é o percentil crítico mínimo que determina que todos os déficits ponderais de $40 \%$ sejam corretamente identificados e que mais da metade dos déficits ponderais de $25 \%$ também o sejam. Senão, vejamos:

a) na referida idade e sexo, é evidente que apenas crianças com peso esperado de $13.150 \mathrm{~g}$ ou mais poderiam suportar $40 \%$ de déficit ponderal sem serem levadas para condições de peso inferior às do percentil $5(8.010 \mathrm{~g})$

b) na mesma idade e sexo, é evidente que apenas crianças com peso esperado de $10.680 \mathrm{~g}$ ou mais poderiam suportar $25 \%$ de déficit ponderal sem igualmente serem levadas para condiçóes de peso inferiores às do percentil 5;

c) ainda na mesma idade e sexo, também é evidente que pesos de $13.150 \mathrm{~g}$ ou mais não são de todo esperados e que pesos de $10.680 \mathrm{~g}$ ou mais são esperados em pouco menos do que metade das crianças. Ou seja, desde que se admita que o padrão utilizado é adequado para a população examinada, evidencia-se que 0 percentil crítico 5 determina ao diagnóstico antropométrico uma sensibilidade de $100 \%$ para os déficits severos (no caso, déficits ponderais de $40 \%$ ) e uma sensibilidade de pouco mais de $50 \%$ para os déficits moderados (no caso, déficits ponderais de $25 \%$ ).

E oportuno salientar que a fixação do percentil crítico mínimo não depende das condições nutricionais da população examinada. Uma vez definidos o que seriam considerados déficits moderados ou severos e definidas as sensibilidades exigidas para déficits como estes, o percentil crítico mínimo passará a depender exclusivamente da variabilidade que a medida antropométrica utilizada apresente em indivíduos normais. Como tal dependerá da faixa etária em questão e do padrão antropométrico adotado.
Do exposto depreende-se que para selecionar o percentil crítico que deverá ser utilizado em um determinado programa de assistência à saúde, os formuladores deste devem percorrer a seguinte seqüência:

a) definir as mínimas especificidade e sensibilidade que almejam do diagnóstico antropométrico;

b) identificar o percentil crítico máximo que garante, em relação à clientela do programa, a mínima especificidade julgada necessária;

c) identificar, a partir do padrão antropométrico adotado, o percentil crítico mínimo que garante a mínima sensibilidade julgada necessária;

d) escolher para percentil crítico o percentil equiidistante aos percentis críticos máximo e mínimo, examinando, ato sucessivo, a especificidade e a sensibilidade efetivamente proporcionadas pelo percentil eleito.

A título de exemplificação, a seqüência proposta foi aplicada à clientela de menores de dois anos do programa de assistência à criança de dois distritos sanitários do Município de São Paulo (Lapa e Butantã):

- a mínima especificidade ainda conveniente ao programa foi estabelecida como a que implicasse que "falsos-positivos" não ultrapassassem metade dos diagnósticos;

- a mínima sensibilidade ainda conveniente ao programa foi estabelecida como aquela que implicasse identificação correta de $100 \%$ dos déficits severos (entendidos como déficits de peso de $40 \%$ ) e $50 \%$ dos déficits moderados (entendidos como déficits de peso de $25 \%$ );

- o percentil 20 de peso foi identificado como o percentil crítico máximo que garantiria a mínima especificidade exigida, pois em uma amostra da clientela em questão demonstrou-se haver $40 \%$ de crianças abaixo do referido percentil, ou seja, o dobro do esperado;

- a partir da distribuição de pesos do padrão "Santo André Classe IV", identificou-se o percentil 5 como o percentil crítico mínimo que garantiria a mínima sen. sibilidade exigida dentro da faixa etária 
MONTEIRO, C. A. Critérios antropométricos no diagnóstico da desnutrição em programas de assistência à criança. Rev. Saúde públ., S. Paulo, 18: 209 - 17, 1984.

em questão (reproduzindo o que já havia sido demonstrado anteriormente para a idade de doze meses);

- na falta de se dispor dos percentis 12 ou 13, escolheu-se o percentil 10 como o percentil crítico a ser utilizado nos dois distritos em questão;

- retornando aos resultados obtidos na amostra examinada e retornando ao padrão antropométrico utilizado, evidenciou-se que o percentil crítico escolhido (percentil 10) capacitaria o diagnóstico antropométrico a:

- trabalhar com especificidade de $90 \%$, significando, na população em questão, contar com pouco menos de $40 \%$ de "falsos-positivos" no total de diagnosticados (na amostra estudada evidenciou-se cerca de $25 \%$ de crianças abaixo do percentil 10);

- identificar corretamente como desnutridas todas as crianças submetidas a déficits ponderais de $40 \%$, $75 \%$ das submetidas a déficits de $25 \%$ e $35 \%$ das submetidas a déficits de $10 \%$.

E oportuno salientar que em distritos sanitários que atendem populações de pior nivel sócio-econômico $\mathrm{e}$, portanto, sujeitas à maior desnutrição, a mesma condição de especificidade mínima poderá ser alcançada com percentis superiores ao percentil 20 . Nestes casos, o percentil crítico escolhido será necessariamente superior ao percentil 10 , o qué permitirá um incremento na sensibilidade, principalmente no referente a déficits menos severos. Por outro lado, em distritos sanitários que atendem população em melhores condiç̋̃es sócio-econômicas, expectativas inversas são esperadas, podendo-se até chegar à situação extrema onde seja impossível contar-se simultaneamente com a especificidade e a sensibilidade julgadas mínimas para o diagnóstico antropométrico, caso em que o mesmo perderia a validade.

\section{CRITÉRIOS ANTROPOMÉTRICOS PROPOSTOS PARA O DIAGNÓSTICO DA DESNUTRIÇĀO E PARA AVALIAÇĀO DA RECUPERAÇÃO NUTRICIONAL.}

Considerando as ponderações preliminares efetuadas quanto ao diagnóstico do estado nutricional infantil por meio da antropometria, considerando as orientaçōes gerais recomendadas quanto ao diagnóstico antropométrico efetuado a nível de unidades sanitárias e considerando também a fisiologia do crescimento e as evidências epidemiológicas disponíveis quanto à história natural da desnutrição em nosso meio, formulamse critérios antropométricos destinados a: 1) diagnosticar a desnutrição e indicar a necessidade da recuperação nutricional; 2) avaliar a eficácia do tratamento instituido; e 3) determinar a alta do tratamento.

\section{1 - CRITÉRIOS ANTROPOMÉTRICOS PARA DIAGNÓSTICO DA DESNUTRIÇÃO E INDICAÇẪO DA RECUPERAÇÃOO NUTRI. CIONAL.}

a) Crianças nos primeiros 6 meses

- Peso atual inferior ao peso crítico para idade e sexo e

- se o peso ao nascer tiver sido inferior ao peso crítico, ganho de peso desde o nascimento inferior ao incremento do peso mediano (condição evidenciada quando a inclinação da curva da criança é inferior à inclinação da curva do percentil 50 ).

OBS: Sendo desconhecido o peso ao nas cer, deve-se considerar o ganho de peso observado entre duas pesadas sucessivas distantes no mínimo de um mês.

b) Crianças de 7 a 24 meses

- Peso atual inferior ao peso crítico para idade e sexo e/ou

- ganho de peso, em duas observações mensais sucessivas, inferior ao incremento correspondente à curva do percentual crítico (condição evidenciada quando a inclinação da curva da criança é inferior à inclinação da curva do percentil crítico). 
MONTEIRO, C. A. Critérios antropométricos no diagnóstico da desnutrição em programas de assistência à criança. Rev. Saúde públ., S. Paulo, 18: $209-17,1984$.

c) Erianças de 25 meses ou mais

- Peso atual inferior ao peso crítico para idade e sexo e

- ganho de peso, em duas observações mensais sucessivas, inferior ao incremento correspondente à curva do percentil crítico.

\section{2- CRITÉRIO ANTROPOMÉTRICO PARA AVALIAR EFICÁCIA DO TRATAMENTO OFERECIDO.}

- Em qualquer idade, ganho de peso igual ou superior ao correspondente ganho previsto a partir da curva do percentil 50 significará que o tratamento está sendo eficaz.

\section{3 - CRITÉRIOS PROPOSTOS PARA ALTA DA RECUPERAÇÃO NUTRICIONAL.}

\section{a) Crianças diagnosticadas nos primeiros 24} meses de vida

- Completar 24 meses de idade e no mínimo 12 meses de seguimento e

- apresentar peso acima do percentil crítico e
- apresentar sucessivos ganhos de peso superiores aos correspondentes incrementos do percentil crítico.

\section{b) Crianças diagnosticades na idade de 25 a} 36 meses

- Completar 36 meses de idade e no mínimo seis meses de seguimento $e$

- apresentar sucessivos ganhos de peso superiores aos correspondentes incrementos do percentil crítico.

c) Crianças diagnosticadas a partir de 36 meses

- Completar no mínimo seis meses de seguimento e

- apresentar sucessivos ganhos de peso superiores aos correspondentes incrementos do percentil crítico.

OBSERVAÇAO: Crianças dos grupos a e b que não se recuperarem nos prazos estipulados serão reinscritas, respectivamente, nos grupos b e c e passarão a atender a especificação de alta destes grupos.

MONTEIRO, C. A. [Anthropometric criteria in the diagnosis of malnutrition in child-assistance programs]. Rev. Saúde públ., S. Paulo, 18: 209 - 17, 1984.

ABSTRACT: Through the examination of the theory which supports the evaluation of nutritional status by mean of anthropometry, and after consideration of characteristics and objectives of children's primary health care, guidelines for the anthropometric-nutritional diagnosis carried out at health centre level are suggested. Further, after consideration of the physiology of growth as well as of evidence on the epidemiology of malnutrition in Brazil, specific criteria for the diagnosis of malnutrition and the needs of nutritional rehabilitation are proposed, with a view to the assessment of the efficacy of treatment and the determination of cure.

UNITERMS: Children, nutritional status. Anthropometry. Malnutrition, diagnosis. 
MONTEIRO, C. A. Critérios antropométricos no diagnóstico da desnutrição em programas de assistência à criança. Rev. Saúde públ., S. Paulo, 18: 209 - 17, 1984.

\section{REFERENNCIAS BIBLIOGRÁFICAS}

1. EVELETH, P. B. \& TANNER, J. M. Worldwide variation in human growth. London, Cambridge University Press, 1976.

2. GOMEZ, F. et al. Mortality in second and third degree malnutrition. $J$. trop. Pediatr. Afr. Child Hlth, 2: 77-83, 1956.

3. MARQUES, R. M. et al. Crescimento e desenvolvimento pubertário em criancas e adolescentes brasileiros. II: altura e peso. São Paulo. Editora Brasileira de Ciências, 1982.

4. MONTEIRO, C. A. et al. Uso da medida do perímetro braquial na detecção do estado nutricional de pré-escolares. Rev. Saúde públ., S. Paulo, 15(supl.): 43-63, 1981.
5. MONTEIRO, C. A. et al. Estudo antropométrico-nutricional de pré-escolares de áreas de baixa renda do Estado de São Paulo, Brasil. Rev. Saúde públ., S. Paulo, 18: 1$18,1984$.

6. MONTHLY VITAL STATISTICS REPORT. (National Center for Health Statistics). Hyattsville, Md, 25(suppl.): 1-22, 1976.

7. UNICEF. Situaçdo mundial da infäncia, 1984. Brasília, DF, 1984.

Recebido para publicação em 23/11/1983. Aprovado para publicaçó em 26/03/1984. 